\title{
Emotional exhaustion in Peruvian university students during the COVID-19 pandemic
}

\author{
Esgotamento emocional em estudantes universitários peruanos durante \\ a pandemia COVID-19
}

\section{Agotamiento emocional en estudiantes universitarios peruanos durante la pandemia de COVID-19}

\author{
Edwin Gustavo Estrada Araoz ${ }^{1}$ iD \\ ${ }^{1}$ Universidad Nacional Amazónica de Madre de Dios, Perú. \\ Autor correspondiente: \\ Edwin Gustavo Estrada Araoz \\ Email: edwin5721@outlook.com \\ Cómo citar: Araoz, E. G. E. (2021). Emotional exhaustion in Peruvian university students during the COVID-19 \\ pandemic. Revista Tempos e Espaços em Educação, 14(33), e16542. http://dx.doi.org/10.20952/revtee.v14i33.16542
}

\begin{abstract}
The COVID 19 pandemic has caused the teaching-learning modality to go from being face-to-face to being virtual, which has forced students to adapt to this new scenario, however, despite the fact that this modality has favorable aspects, it also it has had implications in the socio-emotional aspect. Therefore, the present research aimed to describe the emotional exhaustion in Peruvian students from a private university during the COVID-19 pandemic. The approach was quantitative, the design was non-experimental, and the type was descriptive, transectional. The sample consisted of 265 students, to whom the Emotional Tiredness Scale was applied, an instrument with required levels of validity and reliability. The results found indicate that the students presented high levels of emotional exhaustion (39.4\%) and it was determined that some sociodemographic variables such as sex and age were significantly associated with this condition $(p<0.05)$. It was concluded that it is necessary for the University's Psychology area to detect students with high levels of emotional exhaustion in a timely manner so that they receive treatment and can improve their academic performance and quality of life. On the other hand, students should be encouraged to develop coping strategies for stressful situations, typical of academic life and the context of pandemic.
\end{abstract}

Keywords: COVID-19. Emotional exhaustion. Tiredness. University students.

\section{RESUMO}

A pandemia COVID 19 fez com que a modalidade de ensino - aprendizagem passasse de presencial para virtual, o que obrigou os alunos a se adaptarem a este novo cenário, porém, apesar de esta modalidade apresentar aspectos favoráveis, também teve implicações no aspecto socioemocional. 
Portanto, a presente pesquisa teve como objetivo descrever o esgotamento emocional em estudantes peruanos de uma universidade privada durante a pandemia do COVID-19. A abordagem foi quantitativa, o delineamento não experimental e o tipo descritivo, transversal. A amostra foi composta por 265 alunos aos quais foi aplicada a Escala de Cansaço Emocional, instrumento com níveis exigidos de validade e confiabilidade. Os resultados encontrados indicam que os escolares apresentam níveis elevados de esgotamento emocional $(39,4 \%)$ e determinou-se que algumas variáveis sociodemográficas, como sexo e idade, se associaram significativamente a essa condição $(p<0,05)$. Concluiu-se que é necessário que a área de Psicologia da Universidade detecte em tempo hábil os alunos com altos níveis de exaustão emocional para que recebam tratamento e possam melhorar seu desempenho acadêmico e qualidade de vida. Por outro lado, os alunos devem ser estimulados a desenvolver estratégias de enfrentamento de situações estressantes, próprias da vida acadêmica e do contexto de pandemia.

Palavras-chave: Cansaço. COVID-19. Esgotamento emocional. Estudantes universitários.

\section{RESUMEN}

La pandemia de COVID 19 ha ocasionado que la modalidad de enseñanza - aprendizaje pase de ser presencial a ser virtual, lo cual ha obligado a los estudiantes a adaptarse a este nuevo escenario, sin embargo, a pesar de que dicha modalidad tiene aspectos favorables, también ha tenido implicancias en el aspecto socioemocional. Por ello, la presente investigación tuvo como objetivo describir el agotamiento emocional en los estudiantes peruanos de una universidad privada durante la pandemia de COVID-19. El enfoque fue cuantitativo, el diseño, no experimental y el tipo, descriptivo transeccional. La muestra estuvo conformada por 265 estudiantes a quienes se les aplicó la Escala de Cansancio Emocional, instrumento con niveles de validez y confiabilidad requeridos. Los resultados hallados indican que los estudiantes presentaban altos niveles de agotamiento emocional $(39,4 \%)$ y se determinó que algunas variables sociodemográficas como el sexo y la edad se asociaban de manera significativa con dicho padecimiento $(p<0,05)$. Se concluyó que es necesario que el área de Psicología de la universidad detecte oportunamente a los estudiantes que presenten altos niveles de agotamiento emocional para que reciban un tratamiento y puedan mejorar su desempeño académico y calidad de vida. Por otro lado, se debe fomentar en los estudiantes el desarrollo de estrategias de afrontamiento a situaciones estresantes, propias de la vida académica y del contexto de pandemia.

Palabras clave: Agotamiento emocional. Cansancio. COVID-19. Estudiantes universitarios.

\section{INTRODUCCIÓN}

En el mes de diciembre del año 2019 fueron reportados en China algunos casos de neumonía atípica asociados a un nuevo tipo de coronavirus, al cual se le denominó SARS-CoV-2, causante a su vez de la enfermedad de COVID-19 (Quiroz et al., 2020). Posteriormente y ante el incremento exponencial de casos de dicha enfermedad por todo el mundo, fue categorizada como pandemia en el mes de marzo del año 2020 (Qazi et al., 2020; Buitrago et al., 2021). Este contexto desfavorable produjo una serie de repercusiones en el ámbito sanitario, laboral, social, económico, cultural y desde luego, en la educación (Lizcano \& Arroyave, 2020).

En el Perú, al igual que en otros países, la pandemia de COVID-19 provocó que el Gobierno de turno declare el 15 de marzo del 2020 en estado de emergencia nacional y aislamiento social obligatorio como una medida extrema para evitar la propagación del virus. Por ello, muchas actividades fueron suspendidas, incluso las actividades académicas, tanto en las instituciones educativas básicas como superiores. Sin embargo, para evitar interrumpir el servicio educativo, la modalidad de enseñanza tuvo que migrar de la presencialidad o semipresencialidad a la virtualidad desde el mes de abril del mismo año 2020 (Estrada \& Mamani, 2021). Como es de suponer, esta reforma educativa generó mucha preocupación en los estudiantes, ya que muchos de ellos no se 
encontraban preparados ni mucho menos acostumbrados para afrontar esta nueva forma de aprender (Suárez et al., 2021). No obstante, la prestación del servicio educativo continuó en marcha, ya que era necesario garantizar la continuidad del servicio educativo y no afectar a la comunidad educativa (Toquero, 2020).

Ahora bien, estudiar una carrera universitaria antes de la pandemia resultaba ser muy exigente para los estudiantes y generaba altos niveles de estrés académico debido a las demandas académicas, personales y sociales propias de la antigua normalidad (Estrada et al., 2021a). Así pues, los estudiantes solían participar en distintas actividades académicas propias del mencionado periodo formativo (Garrosa et al., 2017), sin embargo, situaciones específicas como la sobrecarga académica, el limitado tiempo para desarrollar los trabajos, los exámenes, las exposiciones y la excesiva cantidad de horas destinadas a las clases limitaban el tiempo de estudio y la realización adecuada de las responsabilidades académicas (Alfonso et al., 2015).

Posteriormente, durante la pandemia, la situación se volvió más compleja, puesto que los estudiantes tuvieron que afrontar problemas propios de esta modalidad de enseñanza aprendizaje. En primer término, el tecnoestrés y la hiperconexión, producto de la continua exposición de los estudiantes a las computadoras, laptops, tablets y smartphones para participar de las clases sincrónicas y realizar sus demás responsabilidades académicas. Así también, tuvieron que lidiar con las limitaciones en cuanto a accesibilidad y los constantes problemas de conectividad, debido a que muchos de ellos se encontraban en zonas rurales, donde la conexión a internet es pésima. Del mismo modo, se presentaron algunos problemas relacionados directamente con la pandemia, como temer a contagiarse de COVID- 19 y porque también los estudiantes se encontraban con limitaciones económicas ocasionadas porque sus centros de trabajo se cerraron o cambiaron de rubro por las medidas restrictivas impuestas por el gobierno (Estrada \& Gallegos, 2022). Los factores mencionados habrían incrementado los niveles de estrés del estudiantado y favorecería la prevalencia de un fenómeno bastante común en estas épocas: el agotamiento emocional.

El agotamiento emocional fue y aún viene siendo estudiado en el ámbito laboral, no obstante, en el presente estudio será analizado en el contexto académico universitario. En ese sentido, no solo es un padecimiento propio de los trabajadores, sino también de los estudiantes (Fínez \& Morán, 2014). Según la propuesta teórica de Maslach et al. (2001), el agotamiento emocional (provocado por las excesivas demandas académicas) junto a la despersonalización (actitud de indiferencia hacia las actividades académicas) e insatisfacción al logro (conciencia de ineficacia como estudiante), son los tres factores que componen el burnout académico, empero, es el más predominante en el caso de los estudiantes (Topa \& Jurado, 2018; Janurek et al., 2018), ya que los otros dos factores no fueron observados de manera significativa en ellos (Domínguez, 2013; Lledó et al., 2014).

Entonces, el agotamiento emocional se refiere al desgaste psicológico como físico y a la fatiga (Vizoso \& Arias, 2018), es decir, los estudiantes evidencian síntomas somáticos y psicológicos, ya que sienten que no tienen las energías suficientes para realizar las actividades académicas pendientes. Las consecuencias del agotamiento emocional pueden ser reguladas por la capacidad de afrontamiento, el cual es un proceso dinámico que responde a las exigencias objetivas y valoraciones subjetivas que experimentan las personas y puede permitir un manejo apropiado de las emociones frente a una situación percibida como estresante (Barreto \& Salazar, 2021).

Sin embargo, cuando no se cuenta con las adecuadas estrategias de afrontamiento, puede afectar el nivel académico, vocacional, personal y social de los estudiantes. De acuerdo a diversos estudios, el agotamiento emocional se asocia directamente con la ansiedad, la depresión, la ansiedad, la autoculpa, la rumiación, la catastroficación y la ideación suicida (Youssef, 2016; Domínguez \& Merino, 2018; Jiménez et al., 2019). Por otro lado, se ha encontrado asociación inversa con la autoeficacia, la autoestima, el compromiso y la inteligencia emocional (Domínguez, 2018). 
Ahora bien, en el contexto universitario, un estudiante con niveles significativos de agotamiento emocional puede sentir soledad, frustración e indiferencia, lo cual afectaría sus niveles de motivación y la autopercepción de las habilidades y destrezas para el desenvolvimiento de su futura profesión. Además, en casos más complejos, la presencia del agotamiento emocional podría promover el ausentismo, bajo rendimiento académico y a largo plazo puede provocar la deserción universitaria (Rigg et al., 2013).

Como se puede ver, el agotamiento emocional surge de las exigencias académicas y según lo mencionado, puede derivar más adelante en actitudes de cinismo y desinterés por los estudios para finalmente desarrollar creencias de ineficacia personal (Prada et al., 2020). Actualmente, además de las actividades académicas que exigen una sostenida demanda de tiempo y esfuerzo por parte de los estudiantes y el contexto de la pandemia de COVID-19, las instituciones educativas universitarias peruanas se encuentran elevando sus estándares de calidad para concretizar la acreditación de las carreras universitarias (Cabanach et al., 2016), lo cual supone también incrementar las exigencias en búsqueda de una adecuada formación profesional. Ello podría provocar también algún tipo de distanciamiento emocional y cognitivo de los estudiantes hacia el desarrollo de sus responsabilidades académicas, por ello, un abordaje y detección oportuna es fundamental para evitar las repercusiones en la salud física y emocional de los estudiantes así como en su calidad de vida.

Durante el contexto de emergencia sanitaria ocasionada por la pandemia de COVID-19 se realizaron algunas investigaciones acerca de la prevalencia del agotamiento emocional en los estudiantes universitarios. Aunque los resultados no son concluyentes, ya que la realidad educativa universitaria y la forma cómo afrontaron la educación virtual es distinta en las diversas latitudes, muchos estudios concluyeron que existía una prevalencia bastante considerable (Osorio et al., 2020; Barreto \& Salazar, 2021; Salazar et al., 2021; Seperak et al., 2021; Estrada et al., 2021b). Por otra parte, existen estudios que además determinaron que la prevalencia del agotamiento emocional se encontraba asociada de manera significativa a algunas variables sociodemográficas como el sexo (Herrera et al., 2016; Fernández et al., 2017; Barreto \& Salazar, 2021) y la edad de los estudiantes (Bolaños \& Rodríguez, 2016; Aguayo et al., 2019).

Por último, el objetivo de la presente investigación fue describir el agotamiento emocional en los estudiantes universitarios peruanos de una universidad privada durante la pandemia de COVID-19.

\section{METODOLOGÍA}

El enfoque de investigación fue cuantitativo, ya que se realizó la recolección de datos para responder a las preguntas de investigación y contrastar las hipótesis definidas con antelación, haciendo uso de la estadística (Sánchez et al., 2018). Respecto al diseño, fue no experimental, ya que la variable agotamiento emocional no fue manipulada, sino se observó tal como se dio en su entorno y luego se analizó (Hernández \& Mendoza, 2018). En cuanto al tipo, fue descriptivo, puesto que se describieron las propiedades y características de la variable analizada (Bisquerra, 2009).

La población fue conformada por 850 estudiantes de una universidad privada de Puerto Maldonado, Perú, matriculados en el ciclo 2021-I. En cuanto a la muestra, estuvo constituida por 265 estudiantes, cantidad que fue estimada mediante un muestreo probabilístico con un nivel de confianza del $95 \%$ y un nivel de significancia del $5 \%$. De acuerdo a la tabla 1 , del total de estudiantes, el $55,8 \%$ eran mujeres y el $44,2 \%$ eran mujeres. Respecto a la edad, el $53,2 \%$ de los estudiantes tenían entre 16 y 20 años, el 26,4\% tenían entre 21 y 25 años, el 15,5\% tenían entre 26 y 30 años y el 4,9\% tenían de 31 a más años. En cuanto a la carrera profesional, el 35,1\% de los participantes estudiaban Derecho, el 34\% estudiaban Contabilidad y el 30,9\% estudiaban Administración. Por último, con relación a la condición laboral, el $60 \%$ de los estudiantes no trabajaba y el $40 \%$ sí trabajaba. 
Tabla 1. Características de la muestra.

\begin{tabular}{llcc}
\hline Variables sociodemográficas y académicas & & $\mathrm{n}=265$ & $\%$ \\
\hline \multirow{2}{*}{ Sexo } & Masculino & 117 & 44,2 \\
& Femenino & 148 & 55,8 \\
\hline \multirow{3}{*}{ Edad } & De 16 a 20 años & 141 & 53,2 \\
& De 21 a 25 años & 70 & 26,4 \\
& De 26 a 30 años & 41 & 15,5 \\
\multirow{2}{*}{ Carrera profesional } & De 31 a más años & 13 & 4,9 \\
& Administración & 82 & 30,9 \\
\multirow{2}{*}{ Condición laboral } & Contabilidad & 90 & 34,0 \\
& Derecho & 93 & 35,1 \\
\hline
\end{tabular}

La técnica utilizada para la recolección de datos fue la encuesta y el instrumento usado fue la Escala de Cansancio Emocional (Domínguez, 2014), el cual fue estructurado a través del aplicativo Google Forms.

El mencionado cuestionario consta de 10 ítems de factor único y es de tipo Likert (siempre, a veces y nunca). Sus propiedades métricas fueron determinadas a través de los procesos de validez y confiabilidad. Luego de la evaluación de 3 expertos, se determinó que la Escala de Cansancio Emocional posee una adecuada validez de contenido ( $V$ de Aiken=0,802). Asimismo, se halló que la confiabilidad, determinada mediante una prueba piloto a 20 personas, es adecuada $(\alpha=0,819)$.

La recolección de datos se realizó en el mes de junio del año 2021. Para ello se contactó a los estudiantes a través de la aplicación de mensajería Whatsapp, se les explicó el propósito de la investigación y se les envió el link para que accedan y respondan la escala que tuvo una duración de 7 minutos aproximadamente. Posteriormente, el acceso a la aplicación Google Forms fue cerrado una vez que se obtuvieron las 265 respuestas, la información obtenida fue exportada a un archivo de Microsoft Excel y se realizó el proceso calificación tomando en cuenta la escala de valoración.

Para realizar el análisis estadístico se utilizó el Software SPSS versión 22, donde los resultados descriptivos fueron sistematizados a través de tablas de frecuencia y porcentaje. Asimismo, la parte inferencial se realizó utilizando la prueba no paramétrica Chi Cuadrado $\left(\mathrm{X}^{2}\right)$ con el propósito de conocer si el agotamiento emocional de los estudiantes se asociaba de manera significativa con las variables sociodemográficas propuestas.

\section{RESULTADOS}

En la figura 1 se describen los niveles de agotamiento emocional de los estudiantes durante la pandemia de COVID-19. En ese entender, se puede ver que el 39,4\% de ellos presentaban altos niveles de agotamiento emocional, el 36,5\% tenían niveles moderados y el $24,1 \%$ evidenciaban bajos niveles de dicho padecimiento. Se observa así que existe una alta prevalencia en los estudiantes y sería ocasionado principalmente por la hiperconexión, el tecnoestrés, la intermitencia de la conectividad, el temor a contagiarse de COVID-19 y por la precariedad económica por la que atravesaban. 
Figura 1. Niveles de agotamiento emocional

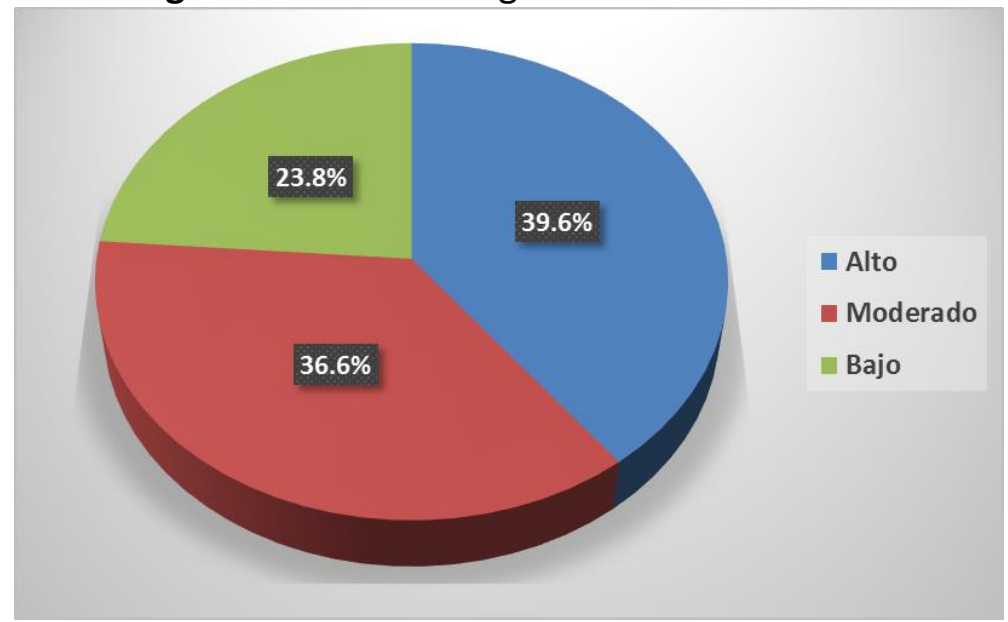

En la tabla 2 se detallan las respuestas a la Escala de Satisfacción con las Clases Virtuales. Así pues, los estudiantes consideran principalmente que no duermen bien por cumplir con sus responsabilidades académicas, se sienten extenuados, tanto física como mentalmente, al culminar sus clases, ya sean sincrónicas o asincrónicas, existen días en el que no cuentan con las energías suficientes para concentrarse y consideran que se les falta tiempo y llegan a sentirse agobiados por la falta de tiempo para desarrollar sus estudios. Por otro lado, es necesario destacar que los estudiantes pocas veces se sentían tristes sin motivo aparente y consideraban que su esfuerzo compensaba sus logros académicos.

Tabla 2. Respuestas a la Escala de Cansancio Emocional.

\begin{tabular}{|c|c|c|c|c|c|c|}
\hline \multirow{2}{*}{ Ítems } & \multicolumn{2}{|c|}{ Siempre } & \multicolumn{2}{|c|}{ A veces } & \multicolumn{2}{|c|}{ Nunca } \\
\hline & $\mathrm{n}$ & $\%$ & $\mathrm{n}$ & $\%$ & $\mathrm{n}$ & $\%$ \\
\hline 1. Los exámenes me producen una tensión excesiva. & 96 & 36,2 & 105 & 39,6 & 64 & 24,2 \\
\hline $\begin{array}{l}\text { 2. Creo que me esfuerzo mucho para lo poco que } \\
\text { consigo. }\end{array}$ & 84 & 31,7 & 95 & 35,8 & 86 & 32,5 \\
\hline $\begin{array}{l}\text { 3. Me siento bajo de ánimo, como triste, sin motivo } \\
\text { aparente. }\end{array}$ & 61 & 23,0 & 112 & 42,3 & 92 & 34,7 \\
\hline 4. Hay días en que no duermo bien a causa del estudio. & 134 & 50,6 & 105 & 39,6 & 26 & 9,8 \\
\hline $\begin{array}{l}\text { 5. Tengo dolor de cabeza y otras molestias que afectan } \\
\text { a mi rendimiento académico. }\end{array}$ & 99 & 37,4 & 109 & 41,1 & 57 & 21,5 \\
\hline $\begin{array}{l}\text { 6. Hay días en que noto más la fatiga y me falta energía } \\
\text { para concentrarme. }\end{array}$ & 105 & 39,6 & 101 & 38,1 & 59 & 22,3 \\
\hline $\begin{array}{l}\text { 7. Me siento emocionalmente agotado por mis } \\
\text { estudios. }\end{array}$ & 99 & 37,4 & 90 & 34,0 & 76 & 28,6 \\
\hline 8. Me siento cansado al final de la jornada de estudio. & 120 & 45,3 & 98 & 37,0 & 47 & 17,7 \\
\hline $\begin{array}{l}\text { 9. Estudiar pensando en los exámenes me produce } \\
\text { estrés. }\end{array}$ & 90 & 34,0 & 110 & 41,5 & 65 & 24,5 \\
\hline $\begin{array}{l}\text { 10. Me falta tiempo y me siento desbordado por los } \\
\text { estudios. }\end{array}$ & 100 & 37,7 & 85 & 32,1 & 80 & 30,2 \\
\hline
\end{tabular}

En la tabla 3 se puede ver que algunas variables sociodemográficas como el sexo y la edad de los estudiantes se asociaban de manera significativa con los niveles de agotamiento emocional que presentaban $(p<0,05)$. No obstante, no se encontraron asociaciones significativas con la carrera profesional y su condición laboral $(p>0,05)$. En virtud a lo expuesto, se pudo determinar que las mujeres más jóvenes presentaban niveles ligeramente superiores de agotamiento emocional en comparación a los demás grupos de contraste. 
Tabla 3. Agotamiento emocional y variables sociodemográficas.

\begin{tabular}{|c|c|c|c|c|c|c|}
\hline \multirow{2}{*}{ Variables } & & \multicolumn{3}{|c|}{ Agotamiento emocional } & \multirow{2}{*}{$x^{2}$} & \multirow{2}{*}{$\begin{array}{c}\mathrm{p}- \\
\text { valor }\end{array}$} \\
\hline & & Alto & Moderado & Bajo & & \\
\hline \multirow{2}{*}{ Sexo } & Masculino & $40(34,2 \%)$ & $45(38,5 \%)$ & $32(27,3 \%)$ & & \multirow{2}{*}{0,012} \\
\hline & Femenino & $65(43,9 \%)$ & $52(35,1 \%)$ & $31(21,0 \%)$ & & \\
\hline \multirow{4}{*}{ Edad } & De 16 a 20 años & $66(46,8 \%)$ & $50(35,5 \%)$ & $25(17,7 \%)$ & & \multirow{4}{*}{0,023} \\
\hline & De 21 a 25 años & $25(35,7 \%)$ & $27(38,6 \%)$ & $18(25,7 \%)$ & & \\
\hline & De 26 a 30 años & $10(24,4 \%)$ & $16(39,0 \%)$ & $15(36,6 \%)$ & & \\
\hline & De 31 a más años & $4(30,8 \%)$ & $4(30,8 \%)$ & $5(38,4 \%)$ & & \\
\hline \multirow{3}{*}{$\begin{array}{l}\text { Carrera } \\
\text { profesional }\end{array}$} & Administración & $31(37,8 \%)$ & $35(42,7 \%)$ & $16(19,5 \%)$ & & \multirow{3}{*}{0,069} \\
\hline & Contabilidad & $28(31,1 \%)$ & $38(42,2 \%)$ & $24(26,7 \%)$ & & \\
\hline & Derecho & $46(49,5 \%)$ & $24(25,8 \%)$ & $23(24,7 \%)$ & & \\
\hline \multirow{2}{*}{$\begin{array}{l}\text { Condición } \\
\text { laboral }\end{array}$} & Trabaja & $42(39,6 \%)$ & $38(35,9 \%)$ & $26(24,5 \%)$ & & \multirow{2}{*}{0,083} \\
\hline & No trabaja & $63(39,6 \%)$ & $59(37,1 \%)$ & $37(23,3 \%)$ & & \\
\hline
\end{tabular}

\section{DISCUSIÓN}

El agotamiento emocional surge como una reacción a la exposición prolongada a factores estresantes, tanto emocionales como interpersonales, en el entorno académico. Ahora bien, dichos factores se han incrementado porque además de las demandas académicas recurrentes, se han incrementado problemas y preocupaciones asociadas a la pandemia. En virtud a lo expuesto, en la presente investigación se describió el agotamiento emocional en los estudiantes peruanos de una universidad privada durante la pandemia de COVID-19 y se determinó qué variables sociodemográficas se encuentran asociadas a su prevalencia.

En primer término, se halló que los estudiantes se caracterizaban por presentar altos niveles de agotamiento emocional y era evidenciado porque no podían dormir bien por cumplir con sus responsabilidades académicas, se sentían extenuados, tanto física como mentalmente, al culminar sus clases virtuales, existían días en el que no contaban con las energías suficientes para concentrarse y llegaban a sentirse agobiados por la falta de tiempo para desarrollar sus trabajos. Asimismo, entre los principales factores que agudizarían este problema estarían la hiperconexión, el tecnoestrés, la intermitencia de la conectividad, el temor a contagiarse de COVID-19 y por la precariedad económica por la que atravesaban.

Los datos expuestos son motivo de preocupación ya que el agotamiento emocional estaría afectando el rendimiento académico de los estudiantes así como su salud física y emocional, tanto en su vida personal, familiar y social. Al respecto, existen investigaciones que coincidieron con los hallazgos descritos, como el trabajo de Barreto y Salazar (2021), quienes realizaron un estudio realizado en estudiantes de las carreras profesionales de psicología, enfermería y medicina en cuatro universidades de la ciudad de Bucaramanga (Colombia) y encontraron que existía una alta incidencia de agotamiento emocional, caracterizado por la presencia de síntomas somáticos (afecciones gástricas, dolores de cabeza, alteraciones en el patrón de sueño) y psicológicos (intranquilidad, tensión, poca concentración, angustia). Del mismo modo, se guarda relación con lo reportado por Fernández et al. (2017) quienes desarrollaron una investigación con estudiantes universitarios españoles y encontraron que dichos estudiantes se caracterizaban por presentar agotamiento emocional, lo que podría ocasionar la disminución de sus expectativas por culminar sus estudios de manera exitosa y estarían menos preparados para afrontar el mundo laboral.

El agotamiento emocional surge como una respuesta al estrés (Maslach \& Leiter, 2016), parte del desequilibrio que existe entre las demandas del contexto académico y los recursos con los que cuenta el estudiante (Sveinsdóttir et al., 2021). Tiene repercusiones negativas, ya que puede afectar las expectativas de los estudiantes así como su madurez profesional e incluso, puede aumentar la probabilidad de deserción y poca preparación para que puedan afrontar el mundo 
laboral (Fontana, 2011). En ese sentido, es menester conocer la incidencia del agotamiento emocional en los estudiantes para poder realizar programas de intervención en los casos que lo requieran (Ríos et al., 2013).

Respecto a las variables sociodemográficas, se determinó que el agotamiento emocional se asociaba de manera significativa con el sexo de los estudiantes $(p<0,05)$. Así pues, las mujeres se caracterizaron por presentar mayores niveles de agotamiento emocional que los varones, lo cual se debería a que ellas suelen exteriorizar las manifestaciones emocionales y fisiológicas producidas por situaciones estresantes y normalmente ellas asumen adicionalmente responsabilidades en sus familias, como el cuidado de los hijos y actividades domésticas (Arias et al., 2019). Lo reportado coincide con los hallazgos de diversos estudios (Lledó et al., 2014; Herrera et al., 2016; Bernable et al., 2016; Fernández et al., 2017; Rodríguez et al., 2019; Estrada \& Gallegos, 2022) que determinaron que existe una mayor prevalencia de agotamiento emocional en las mujeres que en los varones.

Por otro lado, se determinó que el agotamiento emocional se asociaba significativamente con la edad de los estudiantes $(p<0,05)$. De esta manera, los estudiantes más jóvenes se caracterizaron por presentar mayores niveles de agotamiento emocional en comparación a los estudiantes de mayor edad. Ello sería debido a que los estudiantes más jóvenes estarían aprendiendo a utilizar recursos y estrategias de autorregulación de sus emociones para enfrentar los diversos factores estresantes propios de la educación universitaria, sin embargo, los estudiantes con más edad ya habrían adquirido, de manera formal o informal, un repertorio de recursos y estrategias de afrontamiento. El resultado descrito es coherente a algunas investigaciones (Carlotto \& Gonçalves, 2008; Estrada et al., 2017; Aguayo et al., 2019; Estrada et al., 2021b) que determinaron que la edad era una variable sociodemográfica que se asociaba de manera inversa a los niveles de agotamiento emocional, es decir, los estudiantes más jóvenes padecían en mayor medida de dicha enfermedad.

A pesar que en la presente investigación se realizaron hallazgos importantes, es necesario mencionar algunas limitaciones, como la cantidad de estudiantes y su homogeneidad, lo cual no permite generalizar los resultados. Por ello, se recomienda que los futuros estudios incrementa la cantidad de participantes e incluir a estudiantes de otras universidades para que los resultados puedan ser generalizados.

\section{CONCLUSIÓN}

El agotamiento emocional es una forma de respuesta ante situaciones estresantes que se presentan en los diferentes contextos y es caracterizada por la pérdida de las energías, los sentimientos de desgaste físico y psicológico y provoca que los estudiantes incumplan sus responsabilidades académicas y afecten su calidad de vida así como su bienestar psicológico. En ese sentido, en la presente investigación se determinó que los estudiantes universitarios peruanos de una universidad privada se caracterizaban por presentar altos niveles de agotamiento emocional, lo cual sería provocado principalmente por la hiperconexión, el tecnoestrés, la intermitencia de la conectividad, el temor a contagiarse de COVID-19 y por la precariedad económica por la que atravesaban. Asimismo, se determinó que algunas variables sociodemográficas como el sexo y la edad se asociaban de manera significativa a dicho padecimiento $(p<0,05)$.

Frente a ello, es necesario que el área de Psicología de la universidad detecte oportunamente a los estudiantes que presenten altos niveles de agotamiento emocional para que reciban un tratamiento y puedan mejorar su desempeño académico y calidad de vida. Por otro lado, se debe fomentar en los estudiantes el desarrollo de estrategias de afrontamiento a situaciones estresantes, propias de la vida académica y del contexto de pandemia. 
Contribuciones de los autores: Araoz, E. G. E.: concepción y diseño, adquisición de datos, análisis e interpretación de datos, redacción del artículo, revisión crítica de contenido intelectual importante. El autor ha leído y aprobado la versión final del manuscrito.

Aprobación de ética: No aplica.

Agradecimientos: No aplica.

\section{REFERENCIAS}

Aguayo, R., Cañadas, G., Assbaa, L., Cañadas, G., Ramírez, L., \& Ortega, E. (2019). A risk profile of socio-demographic factors in the onset of academic burnout syndrome in a sample of university students. International Journal of Environmental Research and Public Health, 16(5), 707-716. https://doi.org/10.3390/ijerph16050707

Alfonso, B., Calcines, M., Monteagudo, R., \& Nieves, Z. (2015). Estrés académico. EDUMECENTRO, 7(2), 163-178. http://www.revedumecentro.sld.cu/index.php/edumc/article/view/530

Arias, W., Huamaní, J., \& Ceballos, K. (2019). Síndrome de Burnout en profesores de escuela y universidad: un análisis psicométrico y comparativo en la ciudad de Arequipa. Propósitos y Representaciones, 7(3), 72-91.

http://dx.doi.org/10.20511/pyr2019.v7n3.390

Barreto, D., \& Salazar, H. (2020). Agotamiento emocional en estudiantes universitarios del área de la salud. Universidad y Salud, 23(1), 30-39. https://doi.org/10.22267/rus.212301.211

Bernable, E. (2016). Uso del internet y cansancio emocional en estudiantes universitarios. Revista ConCiencia EPG, 1(2), 33-42. https://doi.org/10.32654/CONCIENCIAEPG.1-2.3

Bisquerra, R. (2009). Metodología de la Investigación Educativa. (2a ed.). Madrid: La Muralla S.A.

Bolaños, N., \& Rodríguez, N. (2016). Prevalencia del Síndrome de Burnout académico en el estudiantado de Enfermería de la Universidad de Costa Rica. Revista Enfermería Actual en Costa Rica, 31, 1-19.

http://dx.doi.org/10.15517/revenf.v0i31.24519

Buitrago, F., Ciurana, R., Fernández, M., \& Tizón, J. (2021). Pandemia de la COVID-19 y salud mental: reflexiones iniciales desde la atención primaria de salud española. Atención Primaria, 53(1), 89-101.

https://doi.org/10.1016/j.aprim.2020.06.006

Cabanach, R., Souto, A., \& Franco, V. (2016). Escala de Estresores Académicos para la evaluación de los estresores académicos en estudiantes universitarios. Revista Iberoamericana de Psicología y Salud, 7(2), 41-50.

https://doi.org/10.1016/i.rips.2016.05.001

Carlotto, M., \& Goncalves, S. (2010). Preditores da síndrome de burnout em estudantes universitários. Pensamiento Psicológico, 4(10). https://revistas.javerianacali.edu.co/index.php/pensamientopsicologico/article/view/95

Domínguez, S. (2013). Análisis psicométrico de la escala de cansancio emocional en estudiantes de una universidad privada. Revista Digital de Investigación en Docencia Universitaria, 7(1), 45-55. https://doi.org/10.19083/ridu.7.186

Domínguez, S. (2017). Escala de Cansancio Emocional: estructura factorial y validez delos ítems en estudiantes de una universidad privada. Avances en Psicología, 22(1), 89-97. https://doi.org/10.33539/avpsicol.2014.v22n1.275

Domínguez, S. (2018). Agotamiento emocional académico en estudiantes universitarios: ¿cuánto influyen las estrategias cognitivas de regulación emocional? Educación Médica, 19(2), 96-103.

https://doi.org/10.1016/i.edumed.2016.11.010

Domínguez, S., \& Merino, C. (2018). Cognitive Emotional Regulation Questionnaire-18 en universitarios: Evidencias de validez convergente y discriminante. Revista Iberoamericana de Diagnóstico y Evaluación - e Avaliação Psicológica. RIDEP, 47(2), 171-184, https://doi.org/10.21865/RIDEP47.2.12

Estrada, E., Gallegos, N., \& Mamani, H. (2021b). Burnout académico en estudiantes universitarios peruanos. Apuntes Universitarios, 11(2), 48 - 62. https://doi.org/10.17162/au.v11i2.631

Estrada, E., Mamani, M., Gallegos, N., Mamani, H., \& Zuloaga, M. (2021a). Estrés académico en estudiantes universitarios peruanos en tiempos de la pandemia del COVID-19. Archivos Venezolanos de Farmacología y Terapéutica, 40(1), 88-93. http://saber.ucv.ve/ojs/index.php/rev aavft/article/view/22351

Estrada, E., \& Gallegos, N. (2022). Cansancio emocional en estudiantes universitarios peruanos en el contexto de la pandemia de Covid-19. Educação \& Formação, 7(1), e6759. https://doi.org/10.25053/redufor.v7i1.6759 
Estrada, E., \& Mamani, M. (2021). Competencia digital y variables sociodemográficas en docentes peruanos de educación básica regular. Revista San Gregorio, 1(45), 1-16. http://dx.doi.org/10.36097/rsan.v0i45.1502

Estrada, H., De la Cruz, S., Bahamón, M., Pérez, J., \& Cáceres, A. (2017). Burnout académico y su relación con el bienestar psicológico en estudiantes universitarios. Revista Espacios, 39(15), 7-23.

https://www.revistaespacios.com/a18v39n15/18391507.html

Fernández, E., Liébana, C., \& Morán, C. (2017). Relación entre el sentido de coherencia y el cansancio emocional en estudiantes universitarios. Psychology, Society, \& Education, 9(3), 393-403. https://doi.org/10.25115/psye.v9i3.861

Fínez, M., \& Morán, C. (2014). Resiliencia y autoconcepto: su relación con el cansancio emocional en adolescentes. INFAD Revista de Psicología, 6(1), 289-296. https://doi.org/10.17060/ijodaep.2014.n1.v6.746

Fontana, S. (2011). Estudio preliminar de las propiedades psicométricas de la escala de desgaste emocional para estudiantes universitarios. Revista Argentina de Ciencias del Comportamiento, 3(2), 44-48.

https://doi.org/10.32348/1852.4206.v3.n2.5227

Garrosa, E., Blanco, L., Carmona, I., \& Moreno, B. (2017). How do curiosity, meaning in life, and search for meaning predict college students' daily emotional exhaustion and engagement? Journal of Happiness Studies, 18, 17-40. https://doi.org/10.1007/s10902-016-9715-3

Hernández, R., \& Mendoza, C. (2018). Metodología de la investigación: las rutas cuantitativa, cualitativa y mixta. México: McGraw-Hill.

Herrera, L., Mohamed, L., \& Cepero, S. (2016). Cansancio emocional en estudiantes universitarios. DEDiCA Revista de Educação e Humanidades, 9, 173-191. https://doi.org/10.30827/dreh.v0i9.6888

Janurek, J., Hadi, S., Mojzisch, A., \& Häusser, J. (2018). The association of the 24 hour distribution of time spent in physical activity, work, and sleep with emotional exhaustion. International Journal of Environmental Research and Public Health, 15(9), e1927. https://doi.org/10.3390/ijerph15091927

Jiménez, J., Islas, R., Jiménez, J., Pérez, E., Hernández, M., \& Gonzáles, F. (2019). Emotional exhaustion, burnout, and perceived stress in dental students. Journal of International Medical Research, 47(9), 4251-4259.

https://doi.org/10.1177/0300060519859145

Lizcano, F., \& Arroyave, F. (2020). El ambiente, los desplazamientos y el riesgo cardiovascular en la pandemia por COVID-19. Revista Colombiana de Cardiología, 27(3), 160-165. https://doi.org/10.1016/i.rccar.2020.05.001

Lledó, A., Perandones, T., Herrera, L., \& Lorenzo, G. (2014). Cansancio emocional, autoestima y satisfacción con los estudios en estudiantes universitarios. INFAD Revista de Psicología, 7(1), 161-170.

https://doi.org/10.17060/ijodaep.2014.n1.v7.787

Maslach, C., Schaufeli, W., \& Leiter, M. (2001). Job burnout. Annual Review of Psychology, 52, 397-422.

https://doi.org/10.1146/annurev.psych.52.1.397

Maslach, C., \& Leiter, M. (2016). Understanding the burnout experience: recent research and its implications for psychiatry. World Psychiatry, 15(2), 103-111. https://doi.org/10.1002/wps.20311

Osorio, M., Parrello, S., \& Prado, C. (2020). Burnout académico en una muestra de estudiantes universitarios mexicanos. Enseñanza e Investigación en Psicología, 2(1), 28-37.

https://www.revistacneip.org/index.php/cneip/article/view/86

Prada, R., Navarro, J., \& Domínguez, S. (2020). Personalidad y agotamiento emocional académico en estudiantes universitarios peruanos: un estudio predictivo. Revista Digital de Investigación en Docencia Universitaria, 14(2), e1227. https://doi.org/10.19083/ridu.2020.1227

Qazi, A., Naseer, K., Qazi, J., Alsalman, H., Naseem, U., Yang, S., Hardaker, G., \& Gumaei, A. (2020). Conventional to online education during COVID-19 pandemic: Do develop and underdeveloped nations cope alike. Children and Youth Services Review, 119, e105582. https://doi.org/10.1016/i.childyouth.2020.105582

Quiroz Carrillo, C., Pareja, A., Valencia, E., Enriquez, Y., De Leon, J., \& Aguilar, P. (2020). Un nuevo coronavirus, una nueva enfermedad: COVID-19. Horizonte Médico (Lima), 20(2), e1208.

https://doi.org/10.24265/horizmed.2020.v20n2.11

Rigg, J., Day, J., \& Adler, H. (2013). Emotional exhaustion in graduate students: The role of engagement, self-efficacy and social support. Journal of Educational and Developmental Psychology, 3(2), 138-152.

http://dx.doi.org/10.5539/jedp.v3n2p138 
Ríos, M., Carrillo, C., Sabuco, E., \& Gómez, C. (2013). Agotamiento emocional y síntomas de malestar psíquico en alumnos de enfermería. Revista Iberoamericana de Educación e Investigación en Enfermería, 3(4), 7-13.

Rodríguez, J., Benavides, E., Ornelas, M., \& Jurado, P. (2019). El burnout académico percibido en universitarios; comparaciones por género. Formación Universitaria, 12(5), 23-30. https://dx.doi.org/10.4067/S0718$\underline{50062019000500023}$

Salazar, J., Dolores, E., Valdivia, M., Hernández, M., \& Huerta, I. (2021). Síndrome de burnout en estudiantes de educación superior tecnológica del campus Tierra Blanca en tiempo de covid-19. RIDE Revista Iberoamericana para la Investigación y el Desarrollo Educativo, 11(22), e204. https://doi.org/10.23913/ride.v11i22.896

Sánchez, H., Reyes, C., \& Mejía, K. (2018). Manual de términos en investigación científica, tecnológica y humanística. Lima: Universidad Ricardo Palma.

Seperak, R., Fernández, M., \& Domínguez, S. (2021). Prevalencia y severidad del burnout académico en estudiantes universitarios durante la pandemia por la COVID-19. Interacciones, 7, e199. https://doi.org/10.24016/2021.v7.199

Suárez, J., Bedoya, L., Posada, M., Arboleda, E., Urbina, A., Ramírez, S., Bohórquez, C., \& Ferreira, J. (2021). Percepción de los estudiantes sobre adaptaciones virtuales en cursos de anatomía humana por la contingencia SARS-CoV-2. Academia y Virtualidad, 14(1), 151-168. https://doi.org/10.18359/ravi.5275

Sveinsdóttir, H., Flygenring, B., Svavarsdóttir, M., Thorsteinsson, H., Kristófersson, G., Bernharđsdóttir, J., \& Svavarsdóttir, E. (2021). Predictors of university nursing student's burnout at the time of the COVID-19 pandemic: A cross-sectional study. Nurse Education Today, 106, e105070. https://doi.org/10.1016/i.nedt.2021.105070

Topa, G., \& Jurado, J. (2018). Emotional exhaustion and health complaints as indicators of occupational diseases among civil servants in Spain. Journal of Clinical Medicine, 7(12), e523. https://doi.org/10.3390/jcm7120523

Toquero, C. (2020). Challenges and Opportunities for Higher Education amid the COVID-19 Pandemic: The Philippine Context. Pedagogical Research, 5(4), em0063. https://doi.org/10.29333/pr/7947

Vizoso, C., \& Arias, O. (2018). Resiliencia, optimismo y burnout académico en estudiantes universitarios. European Journal of Education and Psychology, 11(1), 47-59. https://doi.org/10.30552/ejep.v11i1.185

Youssef, F. (2016). Medical student stress, burnout and depression in Trinidad and Tobago. Academic Psychiatry, 40, 69-75. https://doi.org/10.1007/s40596-015-0468-9

Recibido: 31 de Julio de 2021 | Aceptado: 22 de septiembre de 2021 | Publicado: 5 de Octubre de 2021 